\title{
Familial thrombotic risk based on the genetic background of Protein C Deficiency in a Portuguese Study
}

\author{
Teresa Fidalgo ${ }^{1}$, Patrícia Martinho ${ }^{1}$, Ramon Salvado ${ }^{1}$, Licínio Manco ${ }^{2}$, Ana C. Oliveira ${ }^{1}$, Catarina S. \\ Pinto ${ }^{1}$, Elsa Gonçalves ${ }^{1}$, Dalila Marques ${ }^{1}$, Teresa Sevivas ${ }^{1}$, Natália Martins ${ }^{1}$, Maria Letícia Ribeiro ${ }^{1}$ \\ ${ }^{1}$ Serviço de Hematologia Clínica, Centro Hospitalar e Universitário de Coimbra (CHUC), Coimbra, Portugal; ${ }^{2}$ Department of Life Sciences, \\ University of Coimbra, Coimbra, Portugal
}

\begin{abstract}
Introduction: Inherited protein C (PC) deficiency is a well-known risk factor for venous thrombosis (VT). Plasma PC levels are reliable in moderate to severe deficiencies; however, in mildly deficient individuals, the levels may overlap with those considered normal. Genetic studies of PROC, which encodes PC, could help identify carriers; genome-wide association studies (GWAS) have shown that approximately $50 \%$ of phenotypic variation in PC deficiency is caused by the cumulative effects of mutations in several other loci, namely in the PROCR. Patients and methods: With the main objective of determining the genotype/ phenotype correlation in 59 Portuguese individuals from 26 unrelated families with history of thrombosis and repeatedly low/borderline PC plasma levels, we conducted a molecular study by direct sequencing of PROC; PROC promoter haplotypes and PROCR c.4600A >G polymorphism (rs867186), which are known to influence plasma PC concentrations, were also screened. Results: Twelve different PROC mutations were identified, one of them not previously reported, p.Cys105Arg. The mutation types and locations as well as haplotype combinations correlated with the phenotypic severity. The most frequent mutation, p.Arg199X, correlated with the CGTC haplotype and was identified in nine families containing patients with higher numbers of VT episodes. This mutation in homozygous individuals for the CGTC haplotype is a significant risk factor for VT in Portuguese. Conclusion: These genetic family studies allowed the identification of the unknown carriers and individuals at a higher thrombotic risk within each family, thus permitting the evaluation of the need for prophylactic measures, particularly in at-risk situations.
\end{abstract}

Key words hereditary protein $\mathrm{C}$ deficiency; genotype; phenotype; risk factor; venous thrombosis

Correspondence Teresa Fidalgo, Serviço de Hematologia Clínica, CHUC - Unidade de Trombose e Hemostase, Hospital Pediátrico de Coimbra, Av Afonso Romão, Coimbra 3000-602, Portugal. Tel: +351 239480 370; Fax: +351 239717 216; e-mail: teresa. fidalgo@chc.min-saude.pt

Accepted for publication 3 November 2014

doi:10.1111/ejh.12488

Protein $\mathrm{C}$ (PC) is a serine-protease that belongs to the vitamin K-dependent plasma glycoprotein and zymogen group, which plays a major regulatory role in blood coagulation and thrombosis. PC is activated by the thrombin/thrombomodulin complex on the surface of endothelial cells, where it binds with an endothelial PC receptor (EPCR) and proteolytically inactivates factors $\mathrm{Va}(\mathrm{FVa})$ and FVIIIa in the presence of protein $\mathrm{S}$, phospholipids, and calcium (1).

Numerous mutations in the gene coding for PC (PROC) on chromosome $2 \mathrm{q} 14$ lead to several types of PC deficiency (OMIM \#176860) (2): in type I, deficiency levels of both antigen and activity are reduced, whereas in type II, deficiency antigen levels are normal, but one or more functional defects lead to a decreased activity (3). Hereditary PC deficiency is mainly an autosomal dominant inherited disorder, is an established risk factor for venous thrombosis (VT) (4) and arterial ischemic stroke in children (5), and has been associated to arterial thrombosis events at a young age (6). The relative risk increases as the PC concentration falls, with an odds ratio $(\mathrm{OR})$ of $\approx 4$ in individuals with $\mathrm{PC}$ levels of $<65 \%$ (7). PC deficiency appears to be quite rare, with a prevalence of $0.14-0.50 \%$ in the general population and $2.5-6 \%$ in patients with venous thrombosis. Heterozygous individuals have an approximately sevenfold increased risk 
of VT compared with normal individuals (8). The rare form of PC deficiency, severe homozygous (or compound heterozygous), was first described as a cause of neonatal purpura fulminans resulting in rapid death caused by massive venous thrombosis (9, 10). However, a number of adult patients with very low or undetectable plasma PC levels have been found; they exhibit milder symptoms and/or a later onset of the disease (11).

The diagnosis of PC deficiency based on plasma measurements is often difficult in patients with borderline plasma PC levels, due to the fact that not all heterozygotes for a given mutation have a low plasma PC level and because of the large overlap between heterozygotes and non-carriers (3, 7, $12,13)$. It should also be noted that the decrease in plasma $\mathrm{PC}$ concentrations is influenced by age, where the PC concentration level may remain below the adult reference range until adolescence $(14,15)$.

Genetic studies offer a powerful complementary tool, although the alterations found in $P R O C$ are due to either rare mutations or common single nucleotide polymorphisms (SNPs). However, in about $10-30 \%$ of families with PC deficiency, the mutations are not detectable (16).

The SNPs within the $5^{\prime}$ region of PROC, which causally contribute to down transcriptional regulation, show a correlation with low plasma levels of PC and are associated with adverse outcomes in a variety of clinical states. These SNPs, which extend from the promoter region to the end of intron 2, - 1657C/T (rs1799808); -1644A/G (rs1799809); -1479A/T (rs1799810); and -141T/C (rs1158867), show a high degree of linkage disequilibrium (LD) (7, 12, 17). The CGT haplotype of $-1657 \mathrm{C} / \mathrm{T},-1644 \mathrm{~A} / \mathrm{G}$, and $-1479 \mathrm{~A} / \mathrm{T}$ is generally related to lower plasma PC levels than the complementary TAA haplotype and to a higher risk of thrombotic events. The transcriptional efficiency of PROC in these haplotypes has been described as driven by the SNPs at positions -1657 and -1644 . The other two SNPs, $-1479 \mathrm{~A} / \mathrm{T}$ and $-141 \mathrm{~T} / \mathrm{C}$, are in complete LD $\left(r^{2}=1\right)$ and may be involved in regulating splicing events with moderate thrombotic risk (17).

The search for additional polymorphisms, through the genome-wide association studies (GWAS), has been conducted for loci affecting plasma PC levels, and it has been found that $\approx 50 \%$ of the phenotypic variation is caused by the additive effect of SNPs in other genes $(18,19)$. With this in mind, many studies have been carried out, but only SNPs in the EPCR gene (PROCR) located on chromosome 20q11.2 have been robustly found to be associated with the risk of VT in diverse GWAS $(18,20,21)$. The PROCR polymorphism that shows the highest association with PC levels is the c.4600A $>\mathrm{G}$ (rs867186), causing a serine to glycine substitution at amino acid 219 (p.S219G), located in the transmembrane domain of the EPCR protein. This functional variant showed increased soluble EPCR plasma levels, which explains the increase of PC levels in plasma and contributes to about $10 \%$ of their variability $(22,23)$.
PC deficiency can also be found in association with other genetic defects predisposing for VT including the factor $\mathrm{V}$ Leiden (F5L) and prothrombin G20210A gene polymorphisms. It has been shown that individuals with both PC deficiency and F5L have a higher risk of developing thrombosis at a younger age as compared with carriers of PC deficiency alone $(24,25)$. In individuals with PC deficiency, environmental factors (e.g., pregnancy, puerperium, oral contraceptives, surgery, immobilization, and trauma) are cumulative high-risk factors for thrombosis.

To determine the genotype/phenotype correlation of 59 Portuguese individuals, from 26 unrelated families with a history of personal and/or familial thrombosis, who had repeated low or borderline PC plasma levels, we carried out the molecular study and the haplotype analysis for those polymorphisms which are known to influence the plasma PC concentration.

\section{Patients and methods}

This study includes a group of 59 patients of Portuguese origin belonging to 26 apparently unrelated families, diagnosed with PC deficiency between 2002 and 2012, attending the Department of Haematology at the Centro Hospitalar de Coimbra, Portugal.

\section{Clinical description of the probands and their families}

The probands were attended in our Center for thrombophilia screening after the thrombotic event or because of their family history of thrombosis. The clinical characteristics of patients were recorded, through a validated questionnaire, focusing on personal and family history of thrombosis disease and acquired risk. Among the 26 probands, 22 had a personal history of thrombosis: two had had arterial thrombosis; two obstetrical complications; 13 venous thromboembolism (11 lower limbs deep vein thrombosis, one lower limbs deep vein thrombosis with pulmonary embolism and one portal vein thrombosis) and five superficial vein thrombosis. The remaining four probands were asymptomatic and studied because first- and second-degree relatives had had venous thrombosis (The detailed list of clinical data can be found in Table 1).

The mean age of the probands at diagnosis was $39.27 \pm 17.25$ yrs with a sex distribution $17 \mathrm{~F}: 9 \mathrm{M}$; the mean age at the time of the first thrombotic episode was $24.67 \pm 16.94$ yrs; 11 (42\%) had experienced more than one thrombotic episode. Nineteen probands had acquired risk factors: contraceptive drugs, varicose veins, trauma, pregnancy, dyslipidemia, hypertension, and immobilization. Three probands aged $24.33 \pm 7.57$ of whom had more than one thrombotic episode, had no identified risk factors and identified risk factors and were considered as having had an idiopathic deep venous thrombosis (DVT) (Table 1). Of the 26 probands, fourteen $(53.8 \%)$ had a positive family history of VT; an examination of their relatives revealed 33 with PC deficiency, but only 7 (21\%) had had thrombotic episodes. 


\section{Controls}

Fifty-two healthy volunteers acted as a control group for measuring of PC activity/antigen levels of adult's normal range, and for determining the PROC promoter haplotypes and p.S219G genotypes in PROCR. The control group include unrelated individuals from laboratory and medical staff (38F : 14M), without an individual or family history of thrombosis, with a mean age of $33.5 \mathrm{yrs} \pm 11.12$. Absence of thrombotic events or a family history of thrombosis was verified by means of a validated questionnaire. The thrombophilia study was also carried out in the control group subjects.

In accordance with the Helsinki Declaration, informed consent was obtained from patients and controls.

\section{Samples and sample processing}

All samples of thrombophilia patients were taken with a minimum of 3 months after the last episode of thrombosis and off anticoagulant therapy for at least $30 \mathrm{~d}$.

Functional and immunological studies were performed on blood collected into vacuum tubes containing $3.2 \%$ sodium citrate and centrifuged within $15 \mathrm{~min}$ at room temperature for $20 \mathrm{~min}$ at $2500 \mathrm{~g}$; the obtained platelet poor plasma is then separated into aliquots and kept frozen at $-80^{\circ} \mathrm{C}$ until use. The separated plasma was later subjected to the functional thrombophilia tests.

Genomic DNA was extracted from EDTA whole blood by automatic isolation on $\mathrm{iPrep}^{\mathrm{TM}}$ instrument using gDNA Blood Kit (Invitrogen, Carlsbad, CA, USA).

\section{Thrombophilia screening}

The following tests were performed in all probands and their relatives:

1. Functional thrombophilia tests - Antithrombin activity, PC amidolytic activity, protein $\mathrm{S}$ free and detection of lupus anticoagulant run on ACL TOP (Instrumentation Laboratory, Milan, Italy). PC antigen, protein S total were determined by enzyme-linked immunosorbent assay (DG-EIA- Grifols, Corgenix $^{\mathrm{TM}}$, Corgenix, Broomfield, Colorado, USA).

2. Molecular thrombophilia tests - The screening of F5L and the prothrombin 20210A allele was carried out using

Table 1 Clinical data of 26 probands with protein C (PC) deficiency

\begin{tabular}{|c|c|c|c|c|c|}
\hline ID & $\begin{array}{l}\text { Age } \\
\text { (yrs)/gender }\end{array}$ & $\begin{array}{l}\text { Thrombotic } \\
\text { history } \\
\text { (Age of onset) }\end{array}$ & Risk factors & $\begin{array}{l}\text { Thrombotic } \\
\text { episode (age } \\
\text { of relapse) }\end{array}$ & $\begin{array}{l}\text { Number of } \\
\text { kindreds with VT }\end{array}$ \\
\hline PC.1 & $45 / F$ & SVT (35) & Meniscus fracture, immobilization & RT $(39,44)$ & 0 \\
\hline PC.2 & $31 / F$ & DVT(19) & No & $\mathrm{RT}(20,21)$ & 1 \\
\hline PC.3 & $64 / F$ & SVT (44) & Hypertension and diabetes & PE (64) & 0 \\
\hline PC. 4 & $33 / F$ & \multicolumn{3}{|c|}{2 stillbirths $34 / 30$ wks by placental thrombosis } & 0 \\
\hline PC. 5 & $33 / F$ & Asymptomatic $^{1}$ & - & & 1 \\
\hline PC.6 & $24 / F$ & DVT (24) & Oral contraceptive & & 0 \\
\hline PC.7 & $21 / \mathrm{M}$ & SVT (21) & No & RT $(22,23) ;$ DVT (29) & 1 \\
\hline PC.8 & $23 / F$ & DVT; PE (19) & \multicolumn{2}{|l|}{ Oral contraceptive, 2 miscarriages $(18,23)$} & 0 \\
\hline PC.9 & 28/F & DVT (26) & \multicolumn{2}{|l|}{ Oral contraceptive, long trip } & 0 \\
\hline PC.10 & $35 / \mathrm{M}$ & DVT (33) & No & CTPV (33) & 0 \\
\hline PC.11 & $56 / \mathrm{M}$ & DVT (56) & Varicose veins, smoker, and dyslipidemia & SVT (57) & 1 \\
\hline PC. 12 & $26 / F$ & DVT (20) & Oral contraceptive, sedentary lifestyle & DVT (27) & 2 \\
\hline PC.13 & $68 / F$ & DVT (34) & Delivery & & 1 \\
\hline PC.14 & $48 / \mathrm{M}$ & DVT (46) & Surgery varicose veins, obesity, sedentary lifestyle & DVT (47) & 0 \\
\hline PC.15 & $33 / \mathrm{M}$ & SVT (33) & \multicolumn{2}{|l|}{ Varicose veins } & 1 \\
\hline PC.16 & $67 / \mathrm{M}$ & Asymptomatic $^{1}$ & \multicolumn{2}{|l|}{-} & 2 \\
\hline PC.17 & $13 / \mathrm{M}$ & Asymptomatic $^{1}$ & \multicolumn{2}{|l|}{-} & 1 \\
\hline PC.18 & $19 / F$ & DVT (17) & \multicolumn{2}{|l|}{ Oral contraceptive } & 0 \\
\hline PC.19 & $42 / F$ & Stroke (42) & \multicolumn{2}{|l|}{ Dyslipidemia } & 0 \\
\hline PC.20 & $45 / F$ & DVT (20) & Pregnancy, varicose veins & DVT, T (34) & 2 \\
\hline PC.21 & $16 / \mathrm{M}$ & Asymptomatic ${ }^{1}$ & \multicolumn{2}{|l|}{-} & 1 \\
\hline PC.22 & $47 / \mathrm{M}$ & DVT (47) & \multicolumn{2}{|c|}{ Renal transplant patient } & 0 \\
\hline PC.23 & Neonate/F & \multicolumn{3}{|c|}{ Arterial cerebral thrombosis, ischemia, and amputation of the fingers of the right hand } & 1 \\
\hline PC.24 & $23 / F$ & \multicolumn{3}{|c|}{ Recurrent miscarriages } & 0 \\
\hline PC. 25 & $33 / F$ & SVT (19) & Hypertension, varicose veins, obesity & $\mathrm{RT}(22,26,33)$ & 1 \\
\hline PC.26 & 29/F & DVT (26) & \multicolumn{2}{|l|}{ Delivery } & 1 \\
\hline
\end{tabular}

VT, venous thrombosis; DVT, deep vein thrombosis; SVT, superficial vein thrombosis; PE, pulmonary embolism; RT, recurrent superficial vein thrombosis; cavernous transformation of the portal vein (CTPV).

${ }^{1}$ Referred to thrombophilia screening by their family history of VT. 
modified multiplex allele-specific polymerase chain reaction (PCR).

\section{Diagnosis of PC deficiency}

The PC amidolytic activity was determined using a chromogenic end-point assay (HemosILtm Protein C; Instrumentation Laboratory), because it is less prone to interferences (26); although when a low ratio of activity to antigen and in order to detect deficiencies type II, the PC anticoagulant activity was also determined by a coagulation end-point assay using ProClot assay derived from Protac ${ }^{\circledR}$ (Instrumentation Laboratory, Orangeburg, NY, USA) performed in accordance with the manufacturer's instructions.

The adult's normal ranges were established in 52 healthy controls (mean $\pm 2 \mathrm{SD}$ ): PC activity $73-143 \%$ and PC antigen $71-135 \%$. The sample values of children and adolescents were compared with the published pediatrics reference ranges with standardized age groups $(14,15)$.

The diagnosis of potential probands with deficiency of PC was established on the basis of the following criteria: plasma levels of PC below the lower normal range cutoff values in at least two different samples.

\section{PROC and PROCR genotype analysis}

All exons, intronic boundaries, $5^{\prime}$ and $3^{\prime}$ UTRs of the PROC gene were PCR amplified using primers as described previously (27) with the exception of the following primers used for amplification of: (i) PROC promoter SNPs rs1799808, rs1799809, and rs1799810: Prm_5'-CCTCCCCTGCCCGCA GA-3'; Prm_5'-CGTGATTCCTGGGCGATGTATT-3'; (ii) SNP rs1158867: Int1_5'-ACTGCATTCTGGAGCTGCTT; Int1_GAAAGGGCTGTCCCTGTGT; and (iii) PROCR SNP rs867186: SG_5'- GCTCCTACACTTCGCTGGTC; SG_5'GAGCTGAAACTTTCCCTTGC. After amplification, the PCR products for all samples were sequenced on an ABI PRISM 3130 Genetic Analyzer (Applied Biosystems, Foster City, CA, USA) using Big Dye Terminator v1.1 Cycle Sequencing Chemistry (Applied Biosystems), according to protocols recommended by the manufacturer.

DNA samples from patients lacking identified mutations using PCR/direct sequencing were screened using multiplexed ligation-dependent probe amplification (MLPA) method with the Kit P265-B1 PROC (MRC, Amsterdam, the Netherlands).

New sequence variants and amino acid changes were designated according to current nomenclature and the recommendations of the Human Genome Variation Society Accession Number JX030036.

\section{In silico analysis of amino acid changes}

The possible impact of coding sequence changes (amino acid substitutions) on the structure and function of PC was assessed using three bioinformatics tools: Sorting Intolerant From Tolerant (SIFT, http://sift.jcvi.org), Polymorphism Phenotyping-2 (PolyPhen-2, http://genetics.bwh.harvard.edu/ pph2/), and MutationTaster (http://mutationtaster.org/).

\section{Statistical analysis}

For continuous variables, differences between groups were analyzed by a Student's $t$-test or Mann-Whitney $U$-test, depending on the normality of the data. A distribution of haplotypes between the studied groups was analyzed using Fisher's exact test. Tests were assumed significant whenever at 2 -tailed $P<0.05$. These statistical analyses were performed using GraphPad Prism 6.02 for Windows (GraphPad Software, La Jolla, CA, USA, www.graphpad.com).

Allele frequencies estimated by simple counting, HardyWeinberg equilibrium probability values, as well as linkage phase statistical inference from diploid data with the (Bayesian) ELB algorithm and haplotype frequencies, were obtained using the software Arlequin, version 3.01 (Excoffier L, Laval G, Schneider S. Evol Bioinform Online. 2005; http://cmpg.unibe.ch/software/arlequin3/).

\section{Results}

\section{Laboratory hemostasis findings}

Forty-three patients were determined to have plasma PC activity levels below $60 \%$; the remaining 16 patients received oral anticoagulation $(n=4)$ or had slightly decreased values $(60-$ $73 \%$ ) along with family histories that suggested PC deficiency $(n=12)$. Therefore, 57 individuals were classified with type I PC deficiency and two with type IIa deficiency (PC22; PC24) with normal antigen concentrations and reductions in both amidolytic and anticoagulant activities.

These 59 patients with suspected PC deficiency were subjected to PROC gene analysis. Twelve different mutations were identified, of which only one was not previously reported in the Human Gene Mutation Database (HGMD). The majority of these were missense (9/12; Fig. 1) and included p.Cys105Arg (not previously described), p.Pro210Leu, p.Gly215Glu, p.Arg220Gln, p.Gly239Arg, p.Val339Met, p.Pro369Leu, p.Arg394Trp, and p.Trp444Cys, whereas the remaining three were a nucleotide change $(-13 \mathrm{~A}>\mathrm{G})$ at the promoter region, a nonsense mutation (p.Arg199X), and a small deletion (p.Leu212Hisfs*2). No mutations were found in one family with low PC levels. Patients among the $60-73 \%$ of those with PC activity levels overlapping the minimal values of the control group mostly belonged to the group with type 1 missense mutations (Fig. 2).

Screening for other inherited thrombophilias revealed the co-inheritance of heterozygous F5L in five individuals from three families (PC13, PC18, and PC23) and heterozygosity for a prothrombin G20210A variant in three individuals 


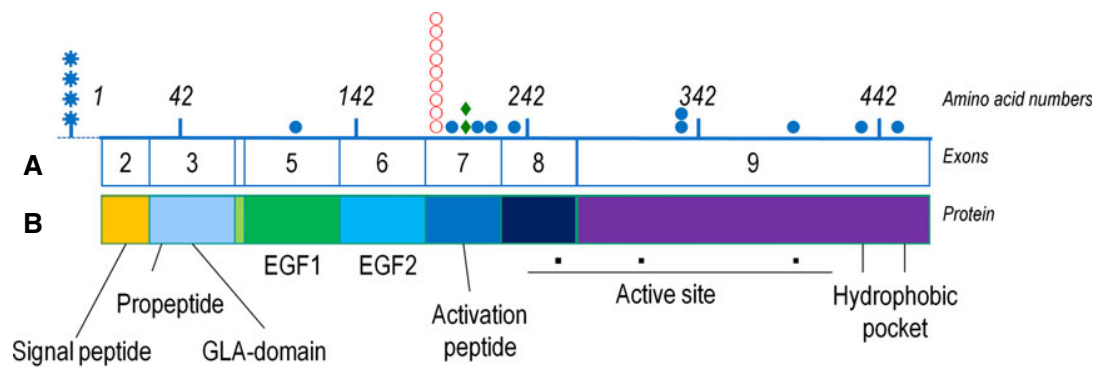

Figure 1 Twelve different mutations were identified in 25 families: promotor $*-13 A>G$; nonsense $\bigcirc$ p.Arg199X; small deletion $>$ p.Leu212Hisfs*2, and missense p.Cys105Arg (not previously described), p.Pro210Leu, p.Gly215Glu, p.Arg220Gln, p.Gly239Arg, p.Val339Met, p.Pro369Leu, p.Arg394Trp, and p.Trp444Cys. The scheme represents their location through of $P R O C$ gene (A) and correspondence of functional or structural domains of protein $(B)$.

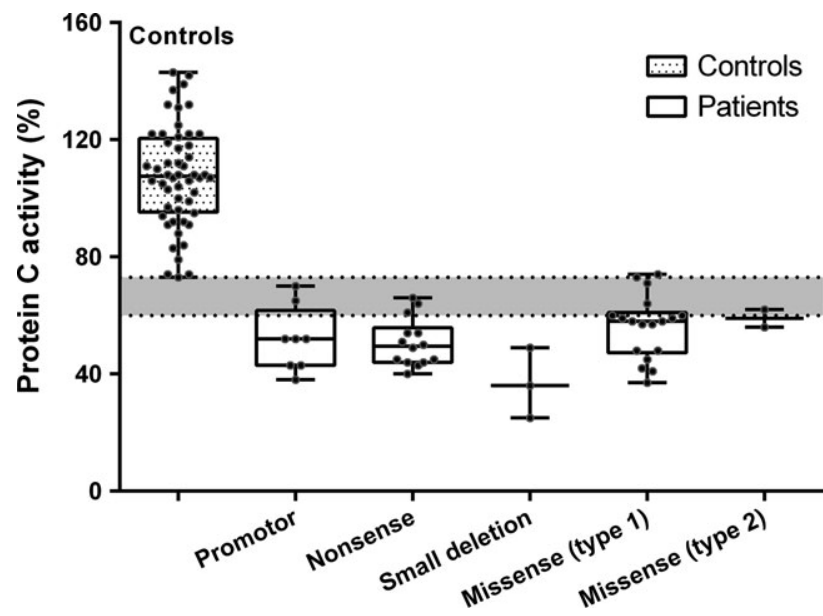

Figure 2 Box-Whisker and dot plots of protein $C(P C)$ activity levels vs. type of mutations. PC activity levels according to the different types of mutation, with each dot representing a single case. The gray zone limits the levels of PC activity $60-73 \%$.

from two families (PC13 and PC14). One patient was homozygous for F5L (PC23) and another was heterozygous for F5L and prothrombin G20210A (PC13.I).

\section{Influence of PROC and PROCR polymorphisms on plasma PC levels}

The distribution of PROC promoter haplotypes based on the SNPs $-1657 \mathrm{C} / \mathrm{T},-1644 \mathrm{~A} / \mathrm{G},-1479 \mathrm{~A} / \mathrm{T}$, and $-141 \mathrm{~T} / \mathrm{C}$ as well as the c.4600A $>\mathrm{G}$ and p.S219G (PROCR) genotypes among the 52 controls and 26 probands is shown in Table 2. Genotype distributions among the control group were in agreement with Hardy-Weinberg equilibrium for all five polymorphisms $(P>0.05)$. Although the CGTC, TAAT, and CAAT PROC promoter haplotypes were observed in both controls and patients, the CGTC frequency was significantly higher among patients than among controls $(P=0.005)$. However, the PROCR c. $4600 \mathrm{~A}>\mathrm{G}$ polymorphism allele frequencies were similar in both groups (minor allele frequency: controls 0.106 vs. patients 0.096; $P=0.713)$.
We further identified the haplotypes associated with each PROC mutation using the four evaluated PROC promoter SNPs (Table 3). The three different haplotypes, CAAT, TAAT, and CGTC, were observed both in normal and mutation carrier chromosomes. Mutations that occur more than once in unrelated probands were always found in the context of a single haplotype. From the twelve identified mutations, the most common nonsense mutation (c.595C $>\mathrm{T}$; chr $=9$ ) and four other missense mutations were associated with the CGTC haplotype; four other different missense mutations were associated with the CAAT haplotype; and the remaining three different types of mutation (one missense, one promoter, and a small deletion) were associated with the TAAT haplotype (Table 3).

The respective influences of the three haplotypes (CGTC, TAAT, and CAAT) and the c.4600A $>$ G genotypes on plasma PC activity were then analyzed in the controls and patients (Fig. 3A). In the control group, the mean PC activity values in the $\mathrm{CG}$ haplotype carrier groups exhibited a downward trend from CGTC/CAAT $=108.7 \%(95 \%$ CI, 93.62-123.8) to CGTC/TAAT $=106 \% \quad(95 \% \quad \mathrm{CI}$, 95.44-116.6) and finally, CGTC/CGTC $=100 \%(95 \% \mathrm{CI}$, 88.5-111.5). In contrast, the PC activity values varied while exhibiting an upward trend from CAAT/CAAT = $103.2 \%(95 \% \mathrm{CI}, 74.93-131.15)$ to $\mathrm{TAAT} / \mathrm{CAAT}=$ $112.9 \%(95 \%$ CI, 97.74-128) and finally, TAAT/ TAAT $=115.6 \% \quad(95 \% \quad$ CI, 104.7-126.5; Fig. 3A). This downward to upward tendency was supported by the significantly different PC activity values between CGTC/ CGTC and TAAT/TAAT carriers $(P=0.0362)$. The mean PC activity values observed in controls regarding the PROCR c.4600A $>\mathrm{G}$ genotypes differed significantly $(P=0.0087)$, with rates of $119.6 \% \quad(95 \%$ CI, $109.7-$ 129.6), with the c.4600AG genotype vs. $104.4 \%$ (95\% CI, 99.08-109.7), and with the c.4600AA genotype (Fig. 3B).

In the patient group, the three PROC haplotypes and $P R O C R$ c.4600A $>\mathrm{G}$ genotypes did not induce significant variability, given the already low levels of PC (Fig. 3A, B). 
Table 2 Distribution of the promoter haplotypes (PROC) and c.4600A $>$ G, p.S219G (PROCR) allele frequencies in controls and 26 probands

\begin{tabular}{lcr}
\hline PROC & $\begin{array}{l}\text { Controls } \\
n(\%)\end{array}$ & $\begin{array}{l}\text { Probands } \\
n(\%)\end{array}$ \\
\hline Haplotype combinations & & $8(30.8)$ \\
CGTC/CGTC & $9(17.3)$ & $8(30.8)$ \\
CGTC/TAAT & $15(28.8)$ & $2(7.7)$ \\
CGTC/CAAT & $7(13.5)$ & $3(11.5)$ \\
TAAT/TAAT & $8(15.4)$ & $3(11.5)$ \\
TAAT/CAAT & $8(15.4)$ & $2(7.7)$ \\
CAAT/CAAT & $5(9.6)$ & $26(100)$ \\
Haplotype frequencies & $52(100)$ & 0.500 \\
CGTC & 0.385 & 0.327 \\
TAAT & 0.375 & 0.173 \\
CAAT & 0.240 & \\
& & \\
\hline PROCR c.4600A>G (p.Ser219G/y) & \\
\hline Genotype & & $21(80.8)$ \\
AA & & $5(19.2)$ \\
AG & $41(78.9)$ & $26(100)$ \\
Allele frequencies & $11(21.1)$ & 0.904 \\
A & $52(100)$ & 0.096 \\
G & 0.894 & 0.713 \\
& 0.106 & \\
\hline
\end{tabular}

1'SNP sequence as follows: $-1657 \mathrm{C} / \mathrm{T},-1644 \mathrm{~A} / \mathrm{G},-1479 \mathrm{~A} / \mathrm{T}$, and $-141 \mathrm{~T} / \mathrm{C}$.

${ }^{*}$ Chi-square test $(\mathrm{O}, \mathrm{E})$ - 2-tailed, $\mathrm{df}=2$, for CGTC haplotype between controls and probands.

${ }^{* *}$ Chi-square test $(\mathrm{O}, \mathrm{E})$ - 2-tailed, $\mathrm{df}=2$, for alleles between controls and probands.

\section{Genetic background of protein C deficiency}

The influence of polymorphisms on plasma PC levels was analyzed in detail on each of the 26 families, as well as potentiates the phenotype of 12 different mutations. The genotype/phenotype correlation was assessed for each of the mutations, their positions, PC activities, and antigen concentrations (Table 4). Only one patient exhibited mutational homozygosity (p.Arg220Gln); all others were heterozygous. The four individuals aged $\leq 17$ yrs (one neonate, one aged $7 \mathrm{yrs}$, and two prepubertal patients aged 13 and 16 yrs) who carried different mutations exhibited significantly lower PC activity levels as compared with older relatives carrying the same mutations (Mann-Whitney $U$-test, $P=0.010$ ). The individuals aged $\leq 17$ yrs and the homozygous subject were analyzed and reported separately because of these differences in activity levels (Table 4).

Promoter mutation: The $-13 \mathrm{~A}>\mathrm{G}$ mutation (La Jolla IV) was shown to abolish 1 of 2 putative HNF-3 binding sites affecting PC promoter transactivation (28). This TAAT haplotype-associated mutation correlated with type I deficiency in eight individuals from four families (Table 4); members

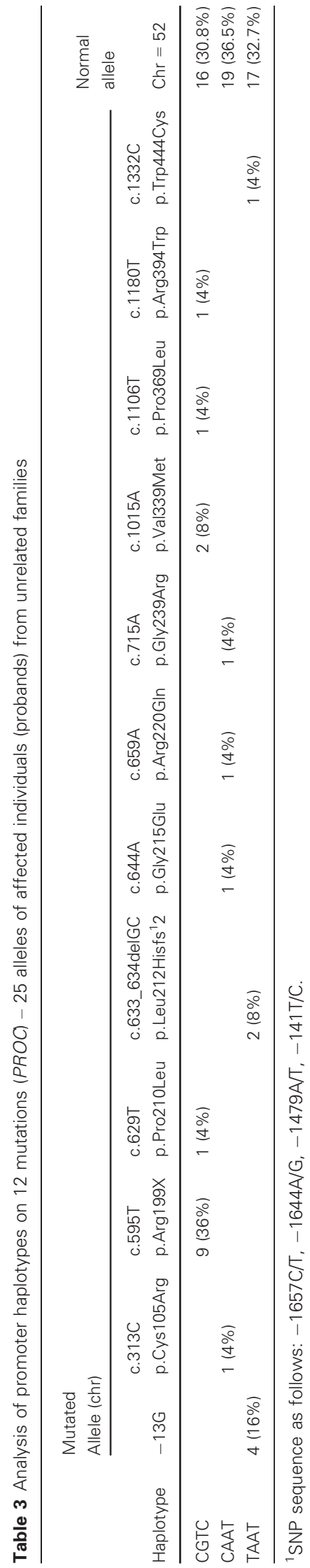



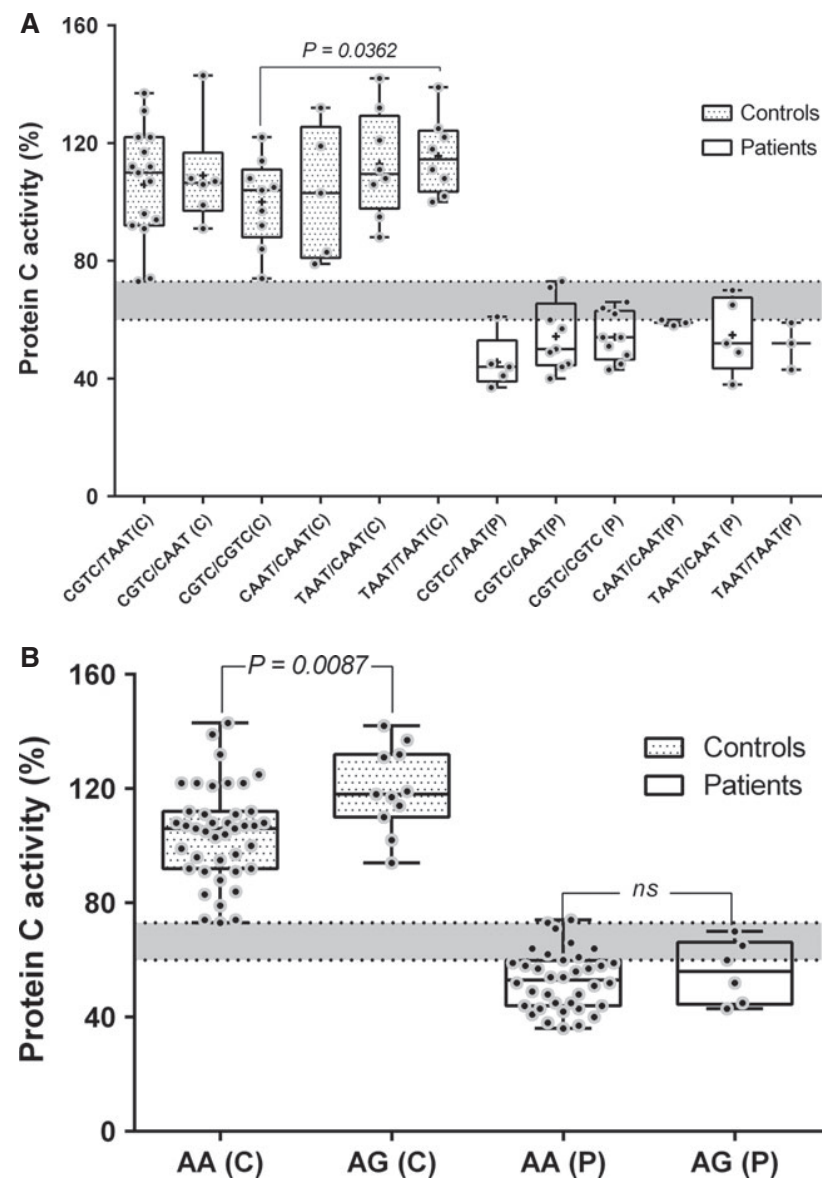

Figure 3 Box-Whisker and dot plots of protein $C(P C)$ activity levels within PROC promoter haplotype combinations (A) and PROCR C. $4600 \mathrm{~A}>\mathrm{G}$ (p.S219G) genotypes (B). (A) PC activity of controls and patients is sorted by six haplotypes combinations and compared to CGTC/CGTC by t-test. (B) PC activity of controls and patients is sorted by c. 4600 AA and AG genotypes and compared by $t$-test. The gray zone limits the levels of PC activity $60-73 \%$. Significance level was 0.05 . If not indicated otherwise comparison was statistically non-significant. (C), controls; (P) patient.

of two of these families were also heterozygous for $P R O C R$ c.4600 A $>\mathrm{G}$ polymorphism. The highest PC activity (70$65 \%)$ and antigen (69-61\%) levels belonged to two relatives who carried the CAAT haplotype in trans and heterozygous for the c.4600 $\mathrm{A}>\mathrm{G}$ polymorphism, one of whom was also a F5L carrier. Two probands (PC.1 and PC.18) experienced VT episodes associated with acquired risk factors (immobilization due to a fractured meniscus and oral contraceptive use), with ages of onset of 35 and $17 \mathrm{yrs}$, respectively. The latter also carried the CGTC haplotype in trans and the c.4600 AG genotype.

Nonsense mutation: The c.595C $>\mathrm{T}$ ( $\mathrm{p} . \operatorname{Arg} 199 \mathrm{X})$ mutation in exon 7 is on a hypermutable $\mathrm{CpG}$ site and introduces a premature termination codon in the connecting dipeptide (Lys198-Arg199) of the light and heavy chains, thus causing disturbances in thrombin-mediated PC activation (29).
This mutation, which is associated with type I PC deficiency, was identified in a heterozygous state in nine probands and nine relatives and was thus the most prevalent mutation among the patients (36\%). All 18 patients carried the PROC promoter CGTC haplotype: nine homozygotes and nine heterozygotes. Among these, 11 of 18 patients (61\%) exhibited early VT complications (age range, 19$44 \mathrm{yrs}$ ), and in one case, pulmonary embolism was also diagnosed (Table 1). Eight of the nine individuals homozygous for CGTC had thrombosis. One proband (PC.13) and his two relatives also had F5L, with one relative additionally carrying the prothrombin G20210A variant although this individual was asymptomatic. Of these three individuals, only the proband was homozygous for the CGTC haplotype. It is also noteworthy that the only three patients from this group (two probands and one relative) who had idiopathic DVT were homozygous for the CGTC haplotype; one of them also carried the c.4600 AG genotype. The fact that the 18 p.Arg199X mutation carrier chromosomes are in complete linkage disequilibrium with a unique CGTC haplotype suggests that at least within the Portuguese population, the c. $595 \mathrm{C}>\mathrm{T}$ nucleotide change occurred once on this progenitor haplotype.

Small deletion: The c.633_634 del GC (Leu212Hisfs*2) mutation is located in the activation cleavage site at Arg211 $\rightarrow$ Leu212, where thrombin cleavage releases a dodecapeptide from the heavy chain; this mutation thus interferes with an indispensable role of the PC structure (1). This mutation was identified in two families (three individuals) with type I PC deficiency and existed with the TAAT haplotype (Tables 3 and 4). Only one individual who was also heterozygous for CGTC haplotype (in trans) developed idiopathic DVT; the other two were asymptomatic (one adult and one child) and presented TAAT haplotype heterozygosity and homozygosity, respectively, thus suggesting a protective effect of this haplotype. None of these individuals carried the PROCR c.4600AG genotype. Besides this study, this deletion has been reported once by another Portuguese group, suggesting that it may have a common ancient origin (30).

Missense mutations: Seven were associated with type I PC deficiency: one in the EGF1 domain, p.Cys105Arg, one in the activation peptide p.Pro210Leu, and five in the serineprotease domain, specifically p.Arg220Gln, p.Gly239Arg, p.Val339Met, p.Pro369Leu, and p.Trp444Cys. The two mutations associated with type II deficiency both occurred in the serine-protease domain: p.Gly215Glu and p.Arg394Trp.

The bioinformatics analyses of these mutations revealed that eight were classified as 'Probably damaging' and one as 'Benign' by PolyPhen, whereas seven were classified as 'Damaging' and two as 'Tolerated' according to SIFT; additionally, the Mutation Taster prediction was 'Disease causing' for seven and 'polymorphism' for two (Table 4). All missense mutations occurred in amino acids that were conserved across species except for p.Arg220Gln and p.Pro369Leu. 


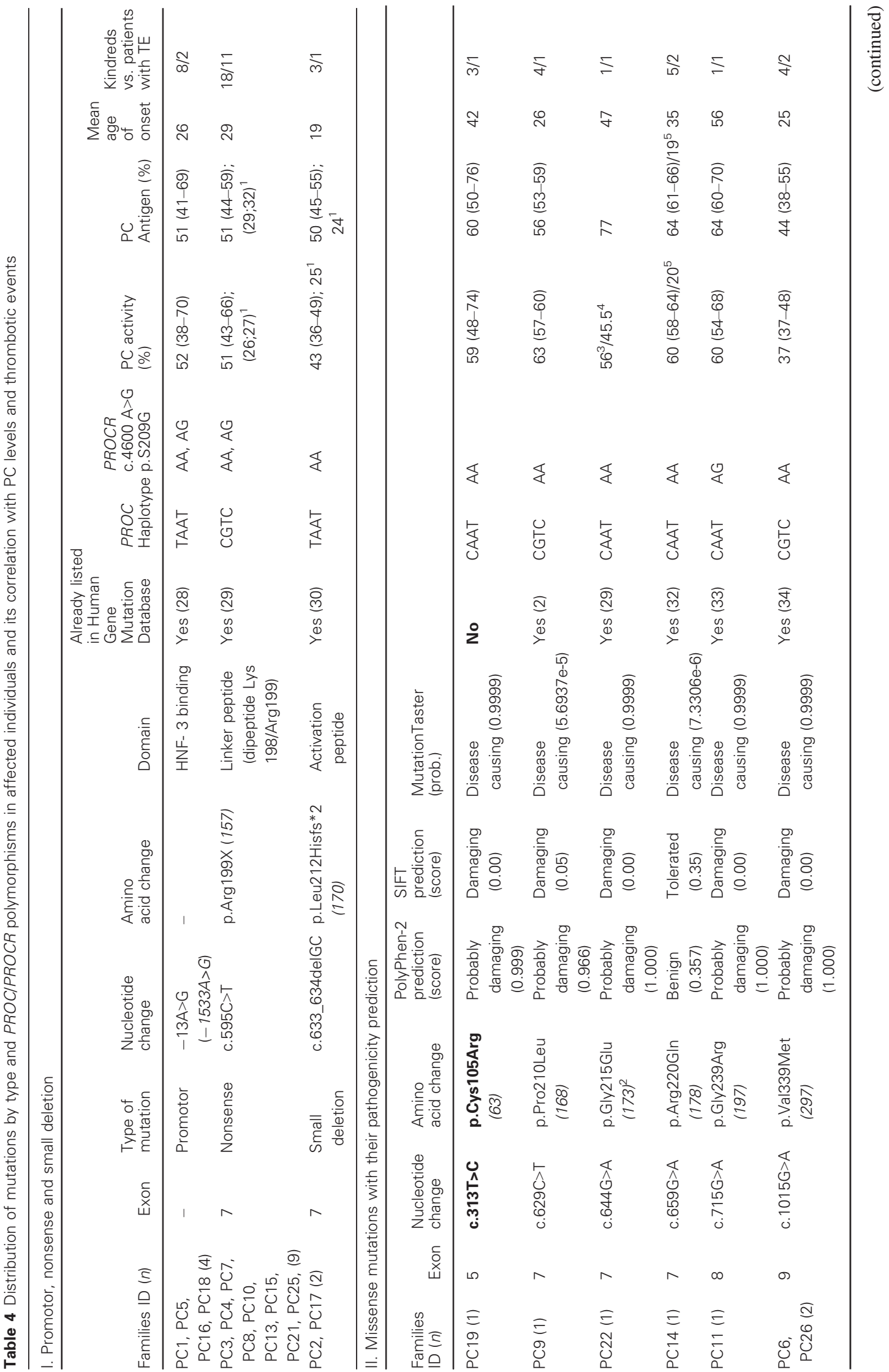




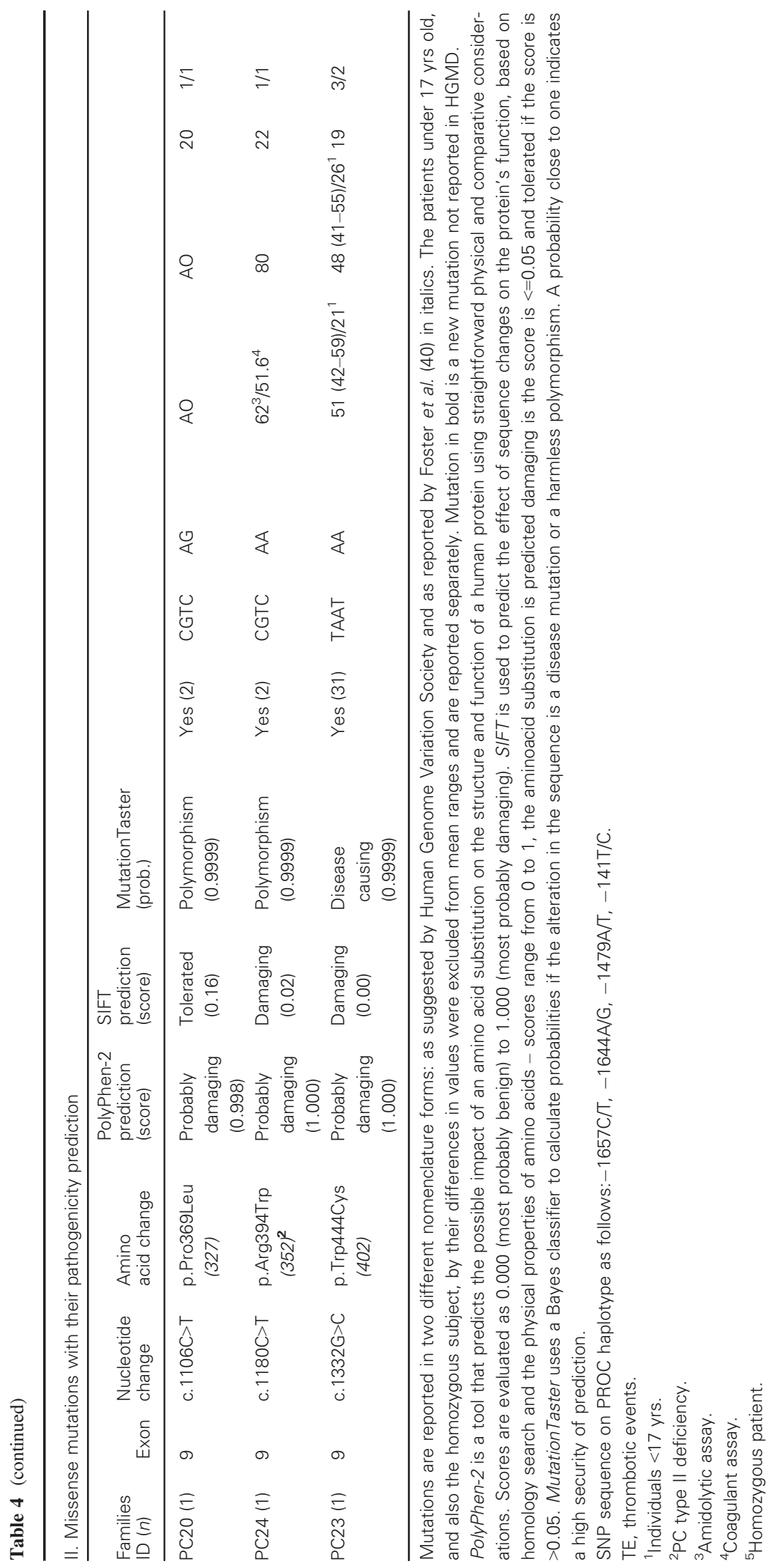


The three deleterious mutations in the catalytic region, p.Gly239Arg, p.Val339Met, and p.Trp444Cys, occurred in four families with type I deficiency and were predicted by PolyPhen, SIFT, and Mutation Taster as 'damaging', 'very high risk', and 'Disease mutation'. These three PC amino acids are highly conserved across orthologs and are thus structurally important. Specifically, in Trp444, the replacement of large tryptophan aromatic ring with a small cysteine hydrophilic side chain leads to tertiary structure destabilization (31). On the other hand, the p.Pro369Leu substitution affects a non-conserved amino acid.

The new mutation p.Cys105Arg was associated with a type I deficiency in one family (three members). The three individuals carried the same promoter PROC haplotype combination of CAAT/TAAT and PROCR genotype c.4600AA; however, only the proband developed thrombosis associated with an acquired risk factor (dyslipidemia). This variant was absent in all 50 healthy individuals, suggesting that it is not a common polymorphism.

The p.Pro210Leu mutation that includes the PROC haplotype combination (CGTC/CAAT) and PROCR genotype c.4600AA was associated with type I deficiency in one family (four members). The proband with a previous risk factor (oral contraceptive use) developed a DVT during a long car trip.

Another mutation found in the proximity of the thrombin activation site was p.Gly215Glu, which was detected with a type II variant as mentioned in the database HGMD (protein C pecs 3) (29). This mutation was found in a renal transplant patient who had been asymptomatic until age $47 \mathrm{yrs}$, the time at which the patient had developed a DVT as a result of an arteriovenous prosthetic device (PC.12). The patient's haplotype combination was CAAT/TAAT and PROCR genotype c.4600AA.

The missense mutation, identified in family PC.14, p.Arg220Gln, was reported as the most recurrent in the database HGMD (32). This locus exhibits three different amino acid substitutions (Gln, Gly, and Pro), with the Gln substitution predicted by both PolyPhen and SIFT as the most 'benign' and 'tolerated', respectively. Four members of family PC.14 were heterozygous and one member was homozygous for this substitution, which caused a PC type I deficiency phenotype. Only two heterozygous subjects had suffered thrombotic events and the homozygous subject, despite having much lower PC levels, remained asymptomatic at 22 yrs of age. However, this family member is quite young and the possibility that she developed thrombosis cannot be ruled out upon exposure to risk factors such as trauma, infection, and pregnancy. The proband, who was heterozygous for the prothrombin G20210A variant, suffered a DVT after surgery for varicose veins. The promoter PROC haplotypes were CAAT/CAAT $(n=3)$, CAAT/TAAT $(n=1)$, and CAAT/CGTC $(n=1)$, and all five individuals carried PROCR c.4600AA genotype.
The mutation p.Gly239Arg in the serine-protease domain (33) was reported in a 60-yr-old man who remained asymptomatic until age $56 \mathrm{yrs}$ (PC.11), the time at which DVT occurred in the presence of the acquired risks of varicose veins, smoking, and dyslipidemia. The promoter PROC haplotype was homozygous CAAT/CAAT with the PROCR genotype c.4600AA. The effect of this deleterious mutation may have been attenuated by the polymorphism genotypes, and DVT may have been triggered by the acquired risks.

The mutation p.Val339Met was present in two families (four individuals). Among all missense mutations, this was associated with the lowest PC values and the two probands had developed DVT at earlier ages (24 and $26 \mathrm{yrs}$ ), thus confirming the deleterious nature of this mutation as described by other authors (34). Besides the contributions of acquired risk factors (oral contraceptive use and delivery) to DVT development, the promoter PROC haplotype CGTC was present in homozygosity $(n=1)$ and in combination with TAAT $(n=3)$ and the PROCR genotype c.4600AA.

The p.Pro369Leu substitution has been associated with a mild phenotype (2). However, this substitution was found in a woman with early-onset VT (20 yrs of age) associated with pregnancy and varicose veins; this patient's promoter PROC haplotype combination was CGTC/TAAT with the PROCR genotype c.4600AG. In this case, CGTC in cis and the c.4600AG genotype may have contributed to the cumulative thrombotic effect of this mutation.

The p.Arg394Trp substitution occurs in the immediate vicinity of the active site S402 (2) and has been associated with a type II deficiency state that was detected in proband PC.14. The carrier of this mutation was a young woman (age, 24 yrs) who had experienced recurrent first-quarter miscarriages; the promoter PROC haplotype was homozygous for CGTC and PROCR genotype c.4600AA. Co-inheritance of the homozygous CGTC haplotype may have had a cumulative effect and contributed to the increased phenotypic severity.

The proband of the family carrying the p.Trp444Cys mutation (31) was an infant who suffered an episode of severe neonatal thrombosis. An arterial cerebral thrombosis during the infant's first day of life followed by subsequent venipuncture for phenobarbital administration led to ischemia requiring amputation to the proximal interphalangeal joints of the second, third, and fourth fingers of the right hand. This family (three individuals) exhibited F5L co-inheritance; the infant and her mother were found to be homozygous and heterozygous, respectively. The mother developed thrombophlebitis during puerperium and the infant's sister remained asymptomatic until $20 \mathrm{yrs}$ of age. It is known that the inheritance of F5L and PROC gene lesions is associated with a cumulative thrombotic risk (10). In this case, the inheritance of a single allele with PROC mutation along with the two F5L alleles was a potentially critical precipitating event in the early-onset thrombotic episodes in the 
affected infant. Curiously, the promoter PROC haplotype was present in both the protective homozygous TAAT/ TAAT (proband and her sister) and heterozygous TAAT/ CGTC (mother) forms; all three individuals carried the PROCR genotype c.4600AA. We may therefore speculate that in the infant's sister, the effect of the Cys444-mutated allele on the PC levels may have been attenuated by the presence of the TAAT haplotype and was thus higher than expected. Regarding the proband child, this haplotype combination (TAAT/TAAT) may have had a positive modulatory effect on the Cys444 mutation and co-inherited homozygous F5L.

Remaining unidentified molecular lesions: Although the PC activity levels of proband PC.12 were low and phenotypically consistent with type I PC deficiency (activity, 14\%; antigen, 17\%), no molecular alterations in PROC were identified in this family (seven individuals) via promoter, exon, and splice-flanking region sequencing. Three of seven individuals experienced VT episodes. The promoter PROC haplotype CGTC was present in both the heterozygous states CGTC/CAAT $(n=3)$ and CGTC/TAAT (CGTA, $n=3$ ) and the homozygous state $(n=1)$ along with the PROCR genotype c.4600AG. The MLPA analysis screening, which is crucial for identifying regions with large molecular defects, did not reveal any alterations. In this case, mutations may have occurred in intronic regions or locus-controlling regions within $P R O C$ or in regions outside of the gene that are important for its expression. mRNA studies may help to elucidate the underlying mechanism.

\section{Genotype/Phenotype analysis}

Assuming that the PC activity correlated inversely with the risk of thrombosis and that genetic risk factors have a cumulative effect, the proportion of thrombotic episodes in the patients was analyzed according to the 12 different mutations and respective haplotypes. The patients were separated according to the occurrence or not of thrombotic episodes to determine the background genetic differences (Fig. 4). It should be noted that patients with PC activity levels ranging from $60 \%$ to $73 \%$ were mostly included in the group without thrombosis. Conversely, in the group of patients with thrombosis, nearly all had PC levels below $60 \%$ and the majority was homozygous for the CGTC haplotype. When the thrombotic events were compared relative to haplotypes (Fig. 5A), patients homozygous for the CGTC haplotype were found to have experienced a significantly higher number of thrombotic events than were other patients $(P=0.0048)$, with a relative risk of $2.3(95 \% \mathrm{CI}=1.5$ 3.6). As shown in Fig. $5 \mathrm{~B}$, the greater proportion of patients homozygous for CGTG (82\%) carried the common nonsense mutation c.595C $>\mathrm{T}$ (p.Arg199X), and this association conferred a relative thrombosis development risk of $2.1(95 \%$ $\mathrm{CI}=1.3-3.2)$.

\section{Discussion}

Thrombosis is a complex disease in which each individual's susceptibility to the disease is determined by the effects of both genetic and acquired risk factors $(23,26)$. PC deficiency is an established risk factor for thrombosis; however, diagnosis based on laboratory plasma level cutoffs is often difficult, and therefore, establishing patients' disease severity and risk of thrombosis becomes challenging. However, family studies are crucial because as proven by several studies, and genetic factors make an approximate $60 \%$ contribution to VT (35).

This study comprised a genotype/phenotype correlation in 26 unrelated families with suspected inherited PC deficiency. Twelve different $P R O C$ gene mutations were identified in 25

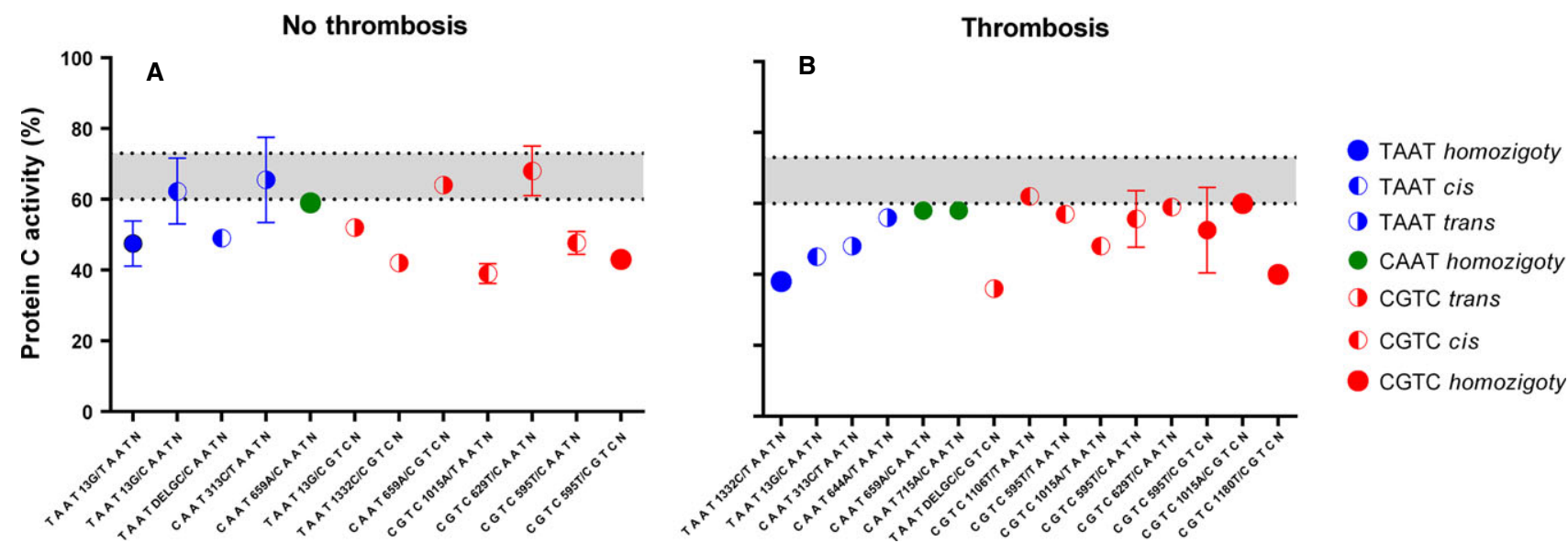

Figure 4 Protein $C(P C)$ activity levels in different mutations and promoter PROC haplotype combinations-distribution by group of patients with thrombosis vs. group of patients without thrombosis. Patients with PC activity levels between $60 \%$ and $73 \%$ (gray zone) were mostly included in the group without thrombosis; conversely in the group of patients with thrombosis nearly all had PC levels below $60 \%$ and the majority was homozygotes for CGTC haplotype. 
Figure 5 Comparison of thrombotic events in patients relative to promoter PROC haplotypes. (A) The number of thrombotic events of patients homozygous for haplotype CGTC had a significantly difference with the other haplotypes when compared by Fisher's exact test; (B) The majority of patients homozygous for CGTG $(82 \%)$ had the nonsense mutation c.595C>T (p.Arg199X). Hmz, homozygous; Htz, heterozygous.
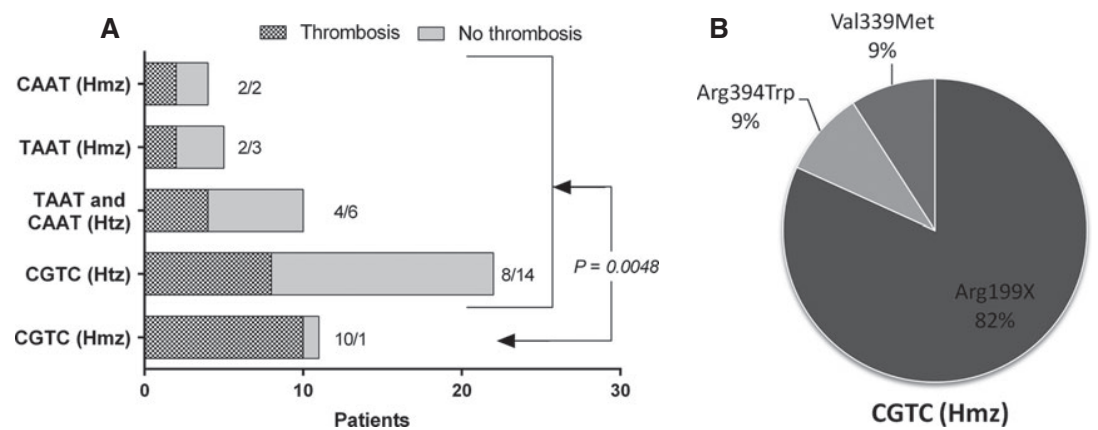

of the 26 families (96\%). The genotype/phenotype correlation was analyzed while considering some known modulating factors such as age, acquired factors, and polymorphisms in the PROC and PROCR genes.

Variability in the plasma PC levels according to age was marked in these families with PC deficiency, which supports the use of age-related normal reference intervals (15). The lowest PC levels observed in individuals $<17$ yrs (two children and two prepubertal individuals) could be explained by a genetic mechanism that underlies the age-related regulation of PROC gene expression. This mechanism acts as a puberty-onset gene switch with an age-related stability element (ASE) that stabilizes gene expression at the pre-puberty, which is then followed by an age-related increase in gene expression (36).

A correlation between $P R O C$ promoter haplotypes and the PROCR c.4600AG polymorphism with respect to plasma PC levels was noted in the 52 controls. The mean PC activity level in individuals homozygous for the PROC CGTC haplotype was approximately $15.6 \%$ less than that of individuals homozygous for the TAAT haplotype. Regarding PROCR c.4600AG, the mean PC activity level in individuals carrying the c.4600G allele was $15 \%$ higher. These results from the control group confirm that the PC levels in healthy individuals are determined by genetic variations. As mentioned in other studies $(7,12,16,17,24)$, the higher CGTC haplotype frequency in the patients in the present study $(P=0.005)$ demonstrated the influence on the thrombotic risk. This association was proven by the higher number of thrombotic episodes experienced by patients homozygous for the CGTC haplotype; conversely, patients homozygous for the TAAT haplotype had the lowest incidence of such events, supporting the notion of a protective effect as suggested previously by other authors (7).

Each of the 12 background genetic mutations justifies the worsening or improvement of a different phenotype (thrombotic risk). These differences are associated with the combinations of mutation types as well as the co-inherited PROC promoter haplotype. Therefore, it was possible to observe a positive modulatory effect on the $-13 \mathrm{~A}>\mathrm{G}$ and $\mathrm{p}$. Leu212Hisfs* 2 mutations conferred by the TAAT haplotype (Fig. 4, Table 4). Similarly, the homozygous patient for
Arg220Gln mutation showed relevant PC levels (19-20\%), what could be explained by a cumulative effect of the benign characteristic of this missense mutation (PolyPhen-2 prediction score $=0.357$ ) associated with the haplotype CAAT/CAAT. In contrast, individuals with mutations that co-inherited the CGTC haplotype such as p.Arg199X and four missense mutations (p.Pro210Leu, p.Val339Met, p.Pro369Leu, and p.Arg394Trp) suffered from a negative modulatory effect. The most frequent p.Arg199X mutation was identified in nine families that contained patients with higher numbers of VT episodes. Regarding missense mutations, we can speculate that the influence of the CGTC haplotype is reflected in the associated earlier mean age of onset relative to that in patients with other mutations (Table 4).

In this small study, the authors did not set out to make new discoveries related to etiological and PC deficiency mechanisms. Large studies with that intent have already been performed and have provided new insights into thrombosis development (37). However, it is necessary to apply these insights into the context of familial studies to determine their usefulness for individual risk prediction (38). We have integrated some of this recent knowledge about the influence of SNPs on plasma PC levels in families with thrombotic events. We performed a genotype/phenotype correlation and noted the thrombotic event severity (type and number) with respect to the background genetic factors and acquired risk factors present in each family. More specifically, the correlation of the mutation type and location as well as the PROC CGTC haplotype and PROCR c.4600AG genotypes with the severity of the phenotype could be verified. For each family, it was possible to establish the genetic background and assess the thrombotic risk associated with each element. The common mutation p.Arg199X correlates with the PROC CGTC haplotype and an increased risk of VT in the Portuguese population. The SNP c.4600A $>\mathrm{G}$ in PROCR may explain differences in the PC plasma levels in individuals with the same mutation. These facts support the hypothesis that next-generation sequencing (NGS) studies will allow the identification of other risk factors; however, this does not exclude the need for correlation genotype/phenotype analyses as these are required to establish family overviews and to elucidate phenotypic discrepancies and 
possibly cases of PC deficiency without identified mutations (23, 37, 39). This study, although geographically limited, illustrates the advantages of identifying the most prevalent mutations in a region.

In conclusion, we demonstrated in this study the importance of establishing the familial thrombotic risk based on the genetic background. Familial mutations, in association with PROC haplotypes, correlated strongly with PC levels as well as the thrombotic episode number and age of onset. Therefore, familial genetic studies of 52 individuals with low or borderline PC plasma levels allowed the identification of suspected carriers and individuals with a higher thrombotic risk that will permit evaluations of the need for prophylactic measures, especially in risky situations.

\section{Addendum}

T. Fidalgo was responsible for study design and coordination and wrote the manuscript; P. Martinho carried out the molecular analysis with contribution of A. Oliveira who also performed data collection of controls; L. Manco contributed to the analysis of data and revision the manuscript; C. Silva Pinto, D. Marques, and E. Gonçalves gave technical support in the laboratory; R. Salvado and T. Sevivas provided clinical support. N. Martins (senior responsible of Haemostasis Unit) and ML Ribeiro (Director of Department) were responsible for revisions of the manuscript.

\section{Acknowledgements}

We like also thank to all the medical and patients who contributed to this study.

\section{Disclosure of conflict of interests}

The authors state that they have no conflict of interest.

\section{References}

1. Esmon CT. The protein C pathway. Chest 2003;124:26-32.

2. Reitsma PH. Protein C deficiency: a database of mutations, 1995 update. Thromb Haemost 1995;73:876-89.

3. Cooper PC, Hill M, Maclean RM. The phenotypic and genetic assessment of protein $\mathrm{C}$ deficiency. Int $\mathrm{J}$ Lab Hematol 2012;34:336-346.

4. Allaart CF, Poort SR, Rosendaal FR, Reitsma PH, Bertina $\mathrm{RM}$, Briët E. Increased risk of venous thrombosis in carriers of hereditary protein $\mathrm{C}$ deficiency defect. Lancet 1993;341:134-8.

5. Kenet G, Lütkhoff LK, Albisetti M, et al. Impact of thrombophilia on risk of arterial ischemic stroke or cerebral sinovenous thrombosis in neonates and children: a systematic review and meta-analysis of observational studies. Circulation 2010;121:1838-47.

6. Mahmoodi BK, Brouwer J-LP, Veeger NJGM, van der Meer J. Hereditary deficiency of protein $\mathrm{C}$ or protein $\mathrm{S}$ confers increased risk of arterial thromboembolic events at a young age: results from a large family cohort study. Circulation 2008;118:1659-67.

7. Aiach M, Nicaud V, Alhenc-Gelas M, Gandrille S, Arnaud E, Amiral J, Guize L, Fiessinger JN, Emmerich J. Complex association of protein $\mathrm{C}$ gene promoter polymorphism with circulating protein $\mathrm{C}$ levels and thrombotic risk. Arterioscler Thromb Vasc Biol 1999;19:1573-6.

8. Koster T, Rosendaal FR, Briët E, van der Meer FJ, Colly LP, Trienekens PH, Poort SR, Reitsma PH, Vandenbroucke JP. Protein $\mathrm{C}$ deficiency in a controlled series of unselected outpatients: an infrequent but clear risk factor for venous thrombosis (Leiden Thrombophilia Study). Blood 1995;85:2756-61.

9. Marlar RA, Montgomery RRBA. Diagnosis and treatment of homozygous protein $\mathrm{C}$ deficiency: report of the working party on homozygous protein $\mathrm{C}$ deficiency of the subcommittee on protein $\mathrm{C}$ and protein $\mathrm{S}$. International Committee of Thrombosis and Haemostasis. J Paediatr 1989;114:528-34.

10. Millar DS, Johansen B, Berntorp E, et al. Molecular genetic analysis of severe protein C deficiency. Hum Genet 2000;106:646-53.

11. Tuddenham EG, Takase T, Thomas aE, Awidi aS, Madanat FF, Abu Hajir MM, Kernoff PB, Hoffbrand AV. Homozygous protein $\mathrm{C}$ deficiency with delayed onset of symptoms at 7 to 10 months. Thromb Res 1989;53:475-84.

12. Spek Ca, Koster T, Rosendaal FR, Bertina RM, Reitsma PH. Genotypic variation in the promoter region of the protein $\mathrm{C}$ gene is associated with plasma protein $\mathrm{C}$ levels and thrombotic risk. Arterioscler Thromb Vasc Biol 1995;15:214-8.

13. Bucciarelli P, Passamonti SM, Biguzzi E, Gianniello F, Franchi F, Mannucci PM, Martinelli I. Low borderline plasma levels of antithrombin, protein $\mathrm{C}$ and protein $\mathrm{S}$ are risk factors for venous thromboembolism. J Thromb Haemost 2012;10:1783-91.

14. Williams MD, Chalmers Ea, Gibson BES. The investigation and management of neonatal haemostasis and thrombosis. Br J Haematol 2002;119:295-309.

15. Flanders MM, Phansalkar AR, Crist RA, Roberts WL, Rodgers GM. Pediatric reference intervals for uncommon bleeding and thrombotic disorders. J Pediatr 2006;149:275-7.

16. Buil A, Soria JM, Souto JC, Almasy L, Lathrop M, Blangero J, Fontcuberta J. Protein C levels are regulated by a quantitative trait locus on chromosome 16: results from the Genetic Analysis of Idiopathic Thrombophilia (GAIT) Project. Arterioscler Thromb Vasc Biol 2004;24:1321-5.

17. Thain KR, Nakada T-A, Boyd JH, Russell JA, Walley KR. A common polymorphism in the $5^{\prime}$ region of the human protein c gene binds USF1. Thromb Res 2012;130:451-7.

18. Tang W, Basu S, Kong X, Pankow JS, Aleksic N, Tan A, Cushman M, Boerwinkle E, Folsom AR. Genome-wide association study identifies novel loci for plasma levels of protein C: the ARIC study. Blood 2010;116:5032-6. 
19. Athanasiadis G, Buil A, Souto JC, Borrell M, López S, Martinez-Perez A, Lathrop M, Fontcuberta J, Almasy L, Soria JM. A genome-wide association study of the Protein C anticoagulant pathway. PLoS One 2011;6:e29168.

20. Pintao MC, Roshani S, de Visser MCH, Tieken C, Tanck MWT, Wichers IM, Meijers JCM, Rosendaal FR, Middeldorp $\mathrm{S}$, Reitsma PH. High levels of protein $\mathrm{C}$ are determined by PROCR haplotype 3. J Thromb Haemost 2011;9:969-76.

21. Dennis J, Johnson CY, Adediran AS, de Andrade M, Heit Ja, Morange P-E, Trégouët DA, Gagnon F. The endothelial protein C receptor (PROCR) Ser219Gly variant and risk of common thrombotic disorders: a HuGE review and meta-analysis of evidence from observational studies. Blood 2012;119:2392-400.

22. Medina P, Navarro S, Estellés A, España F. Polymorphisms in the endothelial protein $\mathrm{C}$ receptor gene and thrombophilia. Thromb Haemost 2007;98:564-9.

23. Morange PE, Tregouet Da. Lessons from genome-wide association studies in venous thrombosis. J Thromb Haemost 2011;9(Suppl 1):258-64.

24. Pomp ER, Doggen CJM, Vos HL, Reitsma PH, Rosendaal FR. Polymorphisms in the protein $\mathrm{C}$ gene as risk factor for venous thrombosis. Thromb Haemost 2009;101:62-7.

25. Navarro S, Medina P, Mira Y, Estellés A, Villa P, Ferrando F, Vayá A, Bertina RM, España F. Haplotypes of the EPCR gene, prothrombin levels, and the risk of venous thrombosis in carriers of the prothrombin G20210A mutation. Haematologica 2008;93:885-91.

26. Baglin T, Gray E, Greaves M, et al. Clinical guidelines for testing for heritable thrombophilia. Br J Haematol 2010;149:209-20.

27. Reitsma PH, Poort SR, Allaart CF, Briët E, Bertina RM. The spectrum of genetic defects in a panel of 40 Dutch families with symptomatic protein $\mathrm{C}$ deficiency type $\mathrm{I}$ : heterogeneity and founder effects. Blood 1991;78:890-4.

28. Spek Ca, Greengard JS, Griffin JH, Bertina RM, Reitsma PH. Two mutations in the promoter region of the human protein $\mathrm{C}$ gene both cause type I protein $\mathrm{C}$ deficiency by disruption of two HNF-3 binding sites. J Biol Chem 1995;270:24216-21.
29. Dávid M, Losonczy H, Sas G, Nagy A, Kutscher G, Meyer M. Identification of mutations in 15 Hungarian families with hereditary protein $\mathrm{C}$ deficiency. Br J Haematol 2000;111:129-35.

30. David D, Ferreira C, Ventura C, Freire I, Moreira I, Gago T. Genetic defects in Portuguese families with inherited protein C deficiency. Thromb Res 2011;128:299-302.

31. Romeo G, Hassan HJ, Staempfli S, et al. Hereditary thrombophilia: identification of nonsense and missense mutations in the protein C gene. Proc Natl Acad Sci USA 1987;84:2829-32.

32. Soria JM, Morell M, Estivill X, Sala N. Recurrence of the PROC gene mutation R178Q: independent origins in Spanish protein C deficiency patients. Hum Mutat 1996;8:71-3.

33. Ireland $\mathrm{H}$, Thompson E, Lane DA, et al. Gene mutations in 21 unrelated cases of phenotypic heterozygous Protein C deficiency and thrombosis. Thromb Haemost 1996;76:867-73.

34. Gandrille S, Aiach M. Identification of mutations in 90 of 121 consecutive symptomatic French patients with a Type I protein C deficiency. Blood 1995;7:2598-605.

35. Germain M, Saut N, Greliche N, et al. Genetics of venous thrombosis: insights from a new genome wide association study. PLoS One 2011;6:e25581.

36. Zhang K, Kurachi S, Kurachi K. Genetic mechanisms of age regulation of protein $\mathrm{C}$ and blood coagulation. $J$ Biol Chem 2002;277:4532-40.

37. Lowe GD. Epidemiology of venous thromboembolism: the need for large (including prospective) studies and meta-analyses. J Thromb Haemost 2012;10:2186-8.

38. Rosendaal FR. Etiology of venous thrombosis: the need for small original studies. J Thromb Haemost 2012;10:2189-90.

39. Oudot-Mellakh T, Cohen W, Germain M, Saut N, Kallel C, Zelenika D, Lathrop M, Trégouët DA, Morange PE, et al. Genome wide association study for plasma levels of natural anticoagulant inhibitors and protein $\mathrm{C}$ anticoagulant pathway: the MARTHA project. PLoS One 2012;6:e255581.

40. Foster DC, Yoshitake S, Davie EW. The nucleotide sequence of the gene for human protein C. PNAS 1985;82:4673-7. 